\title{
Experimental and numerical investigations of flow structure and momentum transport in a turbulent buoyancy-driven flow inside a tilted tube
}

\author{
Citation for published version (APA): \\ Znaien, J. G., Hallez, Y., Moisy, F., Magnaudet, J., Hulin, J. P., Salin, D., \& Hinch, E. J. (2009). Experimental \\ and numerical investigations of flow structure and momentum transport in a turbulent buoyancy-driven flow \\ inside a tilted tube. Physics of Fluids, 21(11), 115102-1/10. [115102]. https://doi.org/10.1063/1.3259972
}

DOI:

$10.1063 / 1.3259972$

Document status and date:

Published: 01/01/2009

\section{Document Version:}

Publisher's PDF, also known as Version of Record (includes final page, issue and volume numbers)

\section{Please check the document version of this publication:}

- A submitted manuscript is the version of the article upon submission and before peer-review. There can be important differences between the submitted version and the official published version of record. People interested in the research are advised to contact the author for the final version of the publication, or visit the DOI to the publisher's website.

- The final author version and the galley proof are versions of the publication after peer review.

- The final published version features the final layout of the paper including the volume, issue and page numbers.

Link to publication

\footnotetext{
General rights

- You may freely distribute the URL identifying the publication in the public portal. follow below link for the End User Agreement:

www.tue.nl/taverne

\section{Take down policy}

If you believe that this document breaches copyright please contact us at:

openaccess@tue.nl

providing details and we will investigate your claim.
}

Copyright and moral rights for the publications made accessible in the public portal are retained by the authors and/or other copyright owners and it is a condition of accessing publications that users recognise and abide by the legal requirements associated with these rights.

- Users may download and print one copy of any publication from the public portal for the purpose of private study or research.

- You may not further distribute the material or use it for any profit-making activity or commercial gain

If the publication is distributed under the terms of Article $25 \mathrm{fa}$ of the Dutch Copyright Act, indicated by the "Taverne" license above, please 


\title{
Experimental and numerical investigations of flow structure and momentum transport in a turbulent buoyancy-driven flow inside a tilted tube
}

\author{
J. Znaien, ${ }^{1}$ Y. Hallez, ${ }^{2}$ F. Moisy, ${ }^{1}$ J. Magnaudet, ${ }^{2}$ J. P. Hulin, ${ }^{1}$ D. Salin, ${ }^{1}$ and E. J. Hinch $^{3}$ \\ ${ }^{1}$ Laboratoire FAST, Université Pierre et Marie Curie-Paris 6, Université Paris-Sud 11, \\ CNRS, F-91405, Bat 502, Campus Universitaire, Orsay F-91405, France \\ ${ }^{2}$ INPT, UPS, IMFT (Institut de Mécanique des Fluides de Toulouse), Université de Toulouse, \\ Allée Camille Soula, F-31400 Toulouse, France and CNRS, IMFT, F-31400 Toulouse, France \\ ${ }^{3}$ DAMTP-CMS, University of Cambridge, Wilberforce Road, Cambridge CB3 OWA, United Kingdom
}

(Received 7 July 2009; accepted 12 October 2009; published online 9 November 2009)

\begin{abstract}
Buoyancy-driven turbulent mixing of fluids of slightly different densities $[$ At $=\Delta \rho /(2\langle\rho\rangle)=1.15$ $\left.\times 10^{-2}\right]$ in a long circular tube tilted at an angle $\theta=15^{\circ}$ from the vertical is studied at the local scale, both experimentally from particle image velocimetry and laser induced fluorescence measurements in the vertical diametrical plane and numerically throughout the tube using direct numerical simulation. In a given cross section of the tube, the axial mean velocity and the mean concentration both vary linearly with the crosswise distance $z$ from the tube axis in the central $70 \%$ of the diameter. A small crosswise velocity component is detected in the measurement plane and is found to result from a four-cell mean secondary flow associated with a nonzero streamwise component of the vorticity. In the central region of the tube cross section, the intensities of the three turbulent velocity fluctuations are found to be strongly different, that of the streamwise fluctuation being more than twice larger than that of the spanwise fluctuation which itself is about $50 \%$ larger than that of the crosswise fluctuation. This marked anisotropy indicates that the turbulent structure is close to that observed in homogeneous turbulent shear flows. Still in the central region, the turbulent shear stress dominates over the viscous stress and reaches a maximum on the tube axis. Its crosswise variation is approximately accounted for by a mixing length whose value is about one-tenth of the tube diameter. The momentum exchange in the core of the cross section takes place between its lower and higher density parts and there is no net momentum exchange between the core and the near-wall regions. A sizable part of this transfer is due both to the mean secondary flow and to the spanwise turbulent shear stress. Near-wall regions located beyond the location of the extrema of the axial velocity $(|z| \geqslant 0.36 d)$ are dominated by viscous stresses which transfer momentum toward (from) the wall near the top (bottom) of the tube. (c) 2009 American Institute of Physics.

[doi:10.1063/1.3259972]
\end{abstract}

\section{INTRODUCTION}

Buoyancy-driven mixing of liquids of different densities is present in many natural flows encountered in the oceans, the atmosphere or in rivers, ${ }^{1,2}$ as well as in industrial processes used in chemical, oil, or environmental engineering. These flows often take place in confined geometries such as tubes or narrow channels (for instance, in artificial wells or in chemical reactors). ${ }^{3-5}$ The corresponding mixing flows strongly differ both from buoyant flows in open geometries and from pressure-driven flows in pipes or channels. A particularly interesting case is provided by long tilted pipes ${ }^{6,7}$ in which, for a zero net axial flow, mixing results from the combined effects of the axial gravity that drives the interpenetration, shear instabilities that induce the transverse mixing, and transverse gravity that moderates it. Here we are specifically interested in steady turbulent mixing flows observed in this geometry at small tilt angles $\theta$ with respect to the vertical in presence of a significant density contrast. ${ }^{8}$

More specifically we perform lock-exchange laboratory experiments and computations, ${ }^{9-11}$ in which each fluid initially fills one-half of the tube length and is set in contact with the other fluid at a time $t=0$ (see Fig. 1). Previous studies of the subsequent interpenetration of the two fluids in the same configuration analyzed macroscopic parameters such as the velocity $V_{f}$ of the displacement fronts ${ }^{7,12}$ or the axial profile of the mean concentration. For instance, the variation of $V_{f}$ with the control parameters of the flow displays nontrivial features in the turbulent mixing regime: $V_{f}$ increases both with the viscosity and the tilt angle while it is almost independent of the density contrast and decreases when the tube diameter increases.

In order to better understand the flow and mixing mechanisms at play, a thorough investigation of the small-scale structure of the velocity and concentration fields is required. A part of this investigation is carried out in the present work using two complementary approaches. On the one hand, local particle image velocimetry (PIV) and laser induced fluorescence (LIF) measurements ${ }^{6}$ are achieved to determine the velocity and concentration fields, respectively. On the other hand, direct numerical simulation ${ }^{7}$ (DNS) is used to investigate the three-dimensional flow structure in the same geom- 


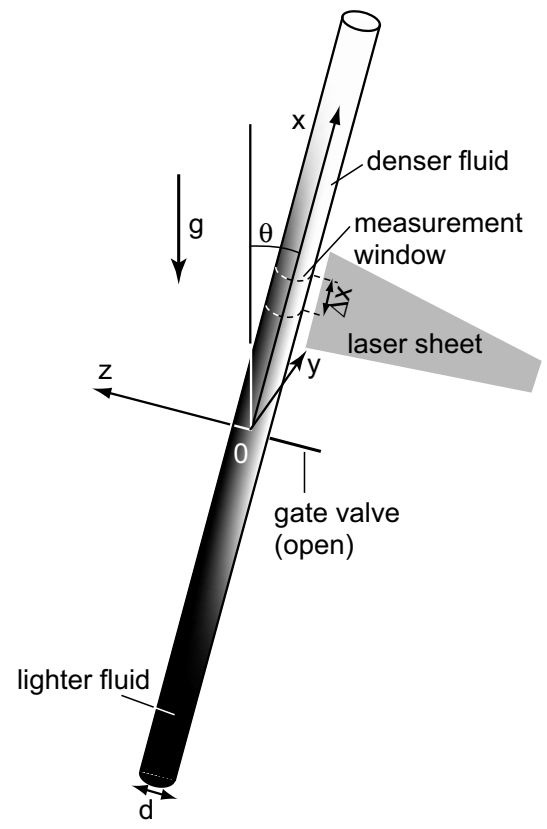

FIG. 1. Schematic of the experimental setup and of the lock-exchange flow induced by the opening of the gate valve. The lighter and denser fluids have the same viscosity $\mu=10^{-3} \mathrm{~Pa} \mathrm{~S}$ and their density contrast $\Delta \rho=23 \mathrm{~kg} \mathrm{~m}^{-3}$ corresponds to an Atwood number At $=1.15 \times 10^{-2}$. The tilt angle is $\theta=15^{\circ}$.

etry. The whole study is performed in a sample test case corresponding to the following values of the control parameters: $\mathrm{At}=\left(\rho_{2}-\rho_{1}\right) /\left(\rho_{2}+\rho_{1}\right)=1.15 \times 10^{-2}, \theta=15^{\circ}$, and $\operatorname{Re}_{t}=\left(\text { At } g d^{3}\right)^{1 / 2} / \nu=950 ; \rho_{2}$ and $\rho_{1}$ are the densities of the denser and lighter fluid and $\nu$ their common kinematic viscosity, respectively. This choice of At and $\theta$ allows us to obtain a well-established turbulent mixing flow while retaining a well-defined stratification due to the transverse gravity component. The measurements are performed far from the displacement fronts, in order to deal with flow regimes close to stationarity.

The computational and experimental approaches provide complementary information. On the one hand, DNS determines the three-dimensional velocity and vorticity fields together with the concentration field but involves large computational times limiting the number of configurations that can be studied and the number of independent numerical "experiments" that can be performed. On the other hand, a large number of laboratory experiments with a long duration can be performed in order to assert the repeatability of the data and the stationarity of the flow regimes. However, these LIF and PIV measurements could only be performed within a vertical diametrical plane so that they do not give access to the spanwise velocity component, nor do they provide the complete flow structure. Moreover the two experimental techniques required separate runs.

The structure of the paper is as follows. After describing the experimental and computational procedures, we discuss the variations of the mean velocity, concentration, and viscous and turbulent stresses across the tube. A mean secondary flow is identified and its origin is quickly discussed. Special attention is paid to the relative magnitude of the mean, turbulent, and viscous momentum transport terms. Finally we analyze the various terms involved in the mean streamwise momentum balance and their relative weight.

\section{FLOW CONFIGURATION, EXPERIMENTAL, AND NUMERICAL METHODOLOGIES}

\section{A. Laboratory experiments}

The experiments are carried out in a long Plexiglas tube (internal diameter: $d=20 \mathrm{~mm}$, length: $L=3.3 \mathrm{~m}$ ) tilted at an angle $\theta=15^{\circ}$ from the vertical. In what follows, the $x$-axis coincides with that of the tube with $x=0$ midway between the two end walls; the $z$-axis is in the vertical diametrical plane whereas the $y$-axis is horizontal (see Fig. 1) with $z=0$ and $y=0$ on the tube axis. We shall frequently refer to directions associated with the $x-, y$-, and $z$-axes as streamwise (or axial), spanwise, and crosswise directions, respectively. Initially, water and a denser $\mathrm{CaCl}_{2}$-water solution fill the lower and upper halves of the tube, respectively, and are separated by a gate valve located at $x=0$.

A 2-mm-thick laser sheet $(\lambda=532 \mathrm{~nm})$ illuminates the vertical $x z$-plane. In order to reduce optical distortions, the tube is surrounded by a transparent enclosure with a square cross section $\left(40 \times 40 \mathrm{~mm}^{2}\right)$ filled with water. In the mixing flow, the optical index contrast due to the density difference between the two fluids is low enough to avoid image distortions.

Once the gate valve is opened, either LIF images or PIV image pairs are obtained at constant time intervals $(0.5$ and $0.25 \mathrm{~s}$, respectively, with a $5 \mathrm{~ms}$ interval between the images of a single PIV pair).

Rhodamin 6G dye at a concentration of $2 \times 10^{-4} \mathrm{~g} / 1$ is added to the lighter fluid to perform LIF measurements; this fluorescent dye has a good compatibility with $\mathrm{CaCl}_{2}$. The procedure used to determine quantitatively the dye concentration is described in Ref. 6 . The local relative volume fraction $c$ of the lighter fluid (referred to as "concentration" in what follows) is then defined as the ratio of the local dye concentration to that of the dye in the pure lighter fluid. The field of view is $120 \times 20 \mathrm{~mm}^{2}$ and its lower side is located at $300 \mathrm{~mm}$ from the gate valve (image resolution of $0.1 \mathrm{~mm} /$ pixel).

Velocity fields are obtained using PIV with both solutions seeded with 1-20 $\mu \mathrm{m}$ diameter fluorescent spheres (PMMA-RhB) and using a $532 \mathrm{~nm}$ notch filter in order to eliminate spurious reflections. Each velocity field contains $160 \times 50$ velocity vectors and the field of view is $64 \times 20 \mathrm{~mm}^{2}$.

\section{B. Computational approach}

The computations are achieved using the JADIM code developed at IMFT to solve the Navier-Stokes equations for an incompressible variable-density fluid without invoking the Boussinesq approximation. Molecular diffusion is neglected, which is appropriate for most liquids, especially those used in the present experiments. To capture properly the discontinuities of the density field at the current fronts, the transport equation for the concentration is advanced by 
means of a first-order time accurate flux corrected technique. The corresponding algorithm is split into three substeps corresponding to the three coordinate axes. For each of them, a Zalesak scheme ${ }^{13}$ is used to compute the advection term. The momentum equation and the incompressibility condition are solved together using a second-order time accurate projection technique. First, the momentum equation is advanced in time using a Runge-Kutta (RK3)/Crank-Nicholson scheme. All spatial derivatives are evaluated by means of secondorder centered schemes. The resulting velocity field is then made divergence-free using a projection step consisting in solving a variable-density pseudo-Poisson equation for the pressure increment. This equation leads to a linear system solved using a Jacobi conjugated gradient technique available in the PETSc library. The overall method is secondorder accurate in space and first-order accurate in time. More technical details and validation tests of the code on configurations close to the present one may be found in Ref. 7.

The computation whose results are described below is carried out in a $20 \mathrm{~mm}$ diameter tube discretized with $32 \times 64$ nodes along the radial and azimuthal directions, respectively. The tube length is progressively increased as time proceeds to ensure that the end walls never influence the two fronts. At the end of the run, the tube length is $3.5 \mathrm{~m}$, and the corresponding grid includes 2816 nodes along the $x$-axis. Convergence tests showed no significant evolution of the solution when the grid resolution was further increased within the cross section. The CPU cost of the computation was about 50000 h using generally 16 and up to 32 processors. The Atwood and Reynolds numbers are identical to those of the experiment. The binary molecular diffusivity is set to zero so that there is, in principle, no diffusion of one fluid into the other. However, in practice, the discretization of sharp density fronts over two to three grid cells induces a finite "numerical" diffusion of the concentration. The corresponding numerical Schmidt number (i.e., the ratio of the kinematic viscosity to the effective diffusivity) has been shown to be of the order of $O\left(10^{3}\right),{ }^{14}$ while the experimental Schmidt number is close to 700 . Therefore, the present numerical technique is expected to reproduce faithfully the laboratory experiment.

\section{Averaging procedure}

In this work, the flow and the momentum transfer are characterized through averaged values of the local concentration and velocity components and through second-order moments of the velocity fluctuations. In experiments as well as in computations, the averages are performed over both time $t$ and streamwise distance $x$; for any variable $f(x, y, z, t)$,

$$
\langle f\rangle(y, z)=\frac{1}{\Delta t} \int_{\Delta t}\left\{\frac{1}{\Delta x} \int_{\Delta x} f(x, y, z, t) d x\right\} d t .
$$

Here, $\Delta x(\Delta t)$ is the spatial (temporal) width of the averaging window. $\Delta t$ corresponds to a time lapse during which the mixing flow can be regarded as stationary. This time lapse is chosen to begin after the transient flow disturbances induced by the front have vanished and ends before the influence of the finite length of the tube is felt in the measurement win- dow. The characteristic length for global variations of the mean flow along $x$ is of the order of the tube length $L$. Hence these variations will not influence the averaged quantities provided $\Delta x \ll L$. With these choices, the averages of the various experimental quantities do not depend on either $x$ or $t$ so that the corresponding derivatives will be neglected.

In the present experiments, the recording begins $200 \mathrm{~s}$ after the transit of the front and lasts for $\Delta t=200 \mathrm{~s}$. Although experimental data are only obtained within one window, the experiments can be easily repeated. The quantities of interest are computed separately for each realization and then averaged. The vertical bars in the plots correspond to the standard deviation of the values obtained in these different realizations.

Computationally, only one run with an "equivalent duration" corresponding to that of the experiments could be achieved, owing to its large CPU cost. However in this run the flow and concentration fields are computed all along the interpenetration zone between the fronts. Therefore several averaging windows (all of length $\Delta x=60 \mathrm{~mm}$ ) located far enough from one another are used to provide several independent data sets. Five different windows located at distances $-250 \leq x \leq+250 \mathrm{~mm}$ have been selected. The streamwise variation of the values obtained in the individual windows did not reveal any definite trend. Therefore, the five data sets have been averaged to obtain the curves displayed below and the vertical bars (when present) indicate the standard deviation of the values from the different windows.

\section{Notation and dimensionless variables}

The streamwise and crosswise velocity components along $x$ and $z$ are denoted as $u$ and $w$, respectively. Since they are located inside the plane of the laser sheet, they are referred to as "in-plane" components. In the same way, the spanwise velocity component $v$ along the $y$-axis is frequently referred to as the "out-of-plane" component. The in-plane terms encountered in the transport equations only involve averages or fluctuations of $u$ and/or $w$, together with derivatives with respect to $z$. They are the only ones we could determine experimentally. The other out-of-plane contributions that involve $v$ and derivatives with respect to $y$ could only be determined from the computation. The streamwise velocity fluctuation $u^{\prime}$ is defined as $u^{\prime}=u-\langle u\rangle$, where $\langle u\rangle$ is the average velocity computed for the same data set. A similar definition is used for the other two fluctuating components $v^{\prime}$ and $w^{\prime}$.

In what follows, most plots and discussions make use of dimensionless variables (characterized with the symbol " $")$. Distances are normalized by the tube diameter $d$, whereas the velocity components $u, v$, and $w$ are normalized by the characteristic velocity $V_{t}=(\text { At } g d)^{1 / 2}$. This velocity scale reflects a balance between buoyancy and inertia and is thus relevant in the present flow regime. ${ }^{15}$ In the present work, $V_{t}$ equals $47.5 \mathrm{~mm} / \mathrm{s}$, which corresponds to a Reynolds number $\operatorname{Re}_{t}=V_{t} d / \nu=950$. 


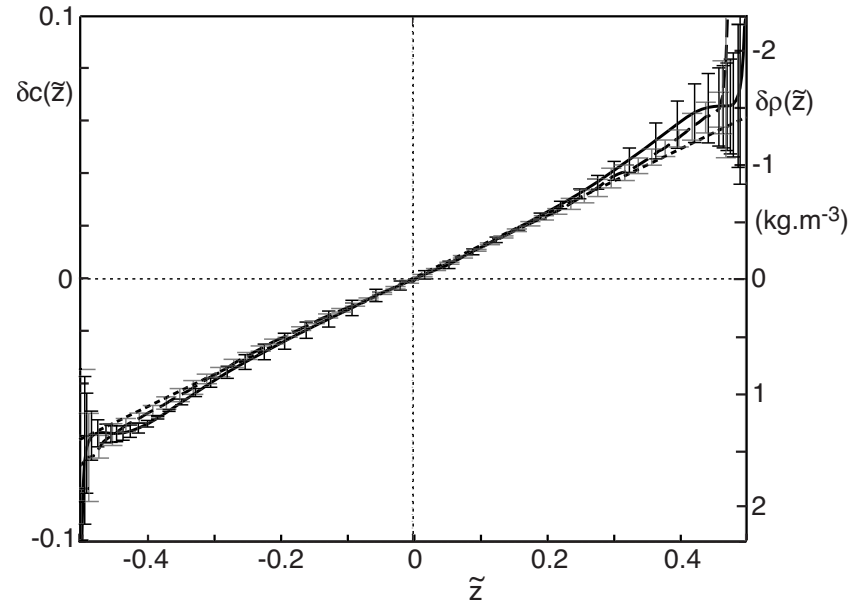

FIG. 2. Relative concentration contrast $\delta c(\widetilde{z})=\langle c(\widetilde{z})\rangle-\langle c(0)\rangle$ as a function of the normalized distance $\tilde{z}=z / d$ to the tube axis in the measurement plane. Right axis: corresponding values of the density contrast $\delta \rho(\widetilde{z})$. Experiments: dashed line. DNS: solid line. Dotted line: linear fit of the DNS data in the central part of the tube. Error bars for experimental data (gray lines): standard deviation of the values obtained in four different realizations. Error bars for numerical simulations (black lines): standard deviation of the values in five windows at different locations above and below the gate valve.

\section{PROFILES OF MEAN CONCENTRATION, VELOCITY, AND TURBULENCE INTENSITIES}

\section{A. Local mean concentration profiles}

Spatial variations of the local concentration $c$ are particularly important because they determine the local buoyancy force that drives the flow (assuming that $\rho$ varies linearly with $c$ ). In a given cross section of the tube, the streamwise component of the gravitational force associated with the streamwise gravity $g_{x}=-g \cos \theta$ may be split into two contributions.

One of them is proportional to the averaged density in the cross section, which itself varies over distances that are large compared to the tube diameter and over long times compared to the local time scale $(d / \text { At } g)^{1 / 2}$, owing to the diffusive spreading of the mean concentration profile. ${ }^{8}$ However this contribution is essentially balanced by the mean pressure gradient along the streamwise direction (which also slowly depends on the streamwise position and time) and therefore has no influence on the local flow structure.

In contrast, there is a second contribution to the streamwise gravitational force that results from the local density differences in the crosswise direction $z$, say $\delta \rho$. We observed that the variations of $\delta \rho$ with the crosswise coordinate $z$ are almost independent of the streamwise position once the turbulent flow is well established far from the end walls of the tube. Hence, in this central portion of the tube, it is relevant to study the flow and concentration characteristics within a cross section without having to consider the streamwise variations of $\delta \rho$ as well as those of the velocity field.

Figure 2 shows the average relative concentration contrast $\delta c(\widetilde{z})=\langle c(\widetilde{z})\rangle-\langle c(0)\rangle$ as a function of $\tilde{z}$. For simplicity, and considering the symmetry of the distribution of $c$ with respect to the diametrical plane $\tilde{z}=0$, we replace the crosssectional average of $c$ by the value $\langle c(0)\rangle$ in this symmetry plane; the averages over $x$ and $t$ are performed as mentioned above. Again, assuming a linear relation between the local density and the concentration, this profile is equivalent to that of the density contrast $\delta \rho(\widetilde{z})=\langle\rho(z)\rangle-\langle\rho(0)\rangle$ since we have

$$
\begin{aligned}
\delta \rho(z) & =\langle\rho(z)\rangle-\langle\rho(0)\rangle \\
& =-(\langle c(z)\rangle-\langle c(0)\rangle) \Delta \rho=-\delta c(z) \Delta \rho,
\end{aligned}
$$

in which $\Delta \rho$ is the difference between the densities of the two pure solutions. The right axis in Fig. 2 displays the density contrast $\delta \rho(\widetilde{z})$.

The collapse of the numerical and experimental data that indicates an excellent agreement between the DNS and the experiments is first worth noting. In both cases, the variation of $\delta c$ with $\tilde{z}$ is linear up to the vicinity of the wall (i.e., for $|z| z 0.45)$ : A dotted line fitted with the data in the region $|\bar{z}| \leq 0.2$ is shown for comparison. In the near-wall region, the variability of the measurements increases rapidly as the wall is approached, preventing an accurate determination of the behavior of $\delta c$ there.

The overall variation of $\delta c(\widetilde{z})$ across the tube diameter is $\delta c(d / 2)-\delta c(-d / 2) \simeq 0.15$, a low value which confirms the efficiency of the transverse turbulent mixing in this flow regime. Moreover, the standard deviation of values of $\delta c(z)$ taken from different realizations does not exceed $\pm 5 \%$ outside the wall region (i.e., for $|\bar{z}| \leq 0.45$ ). This reproducibility allows for meaningful interpretations combining experimental estimates of the buoyancy force derived from these concentration profiles and terms of the momentum balance equation deduced from velocity measurements in distinct experiments.

\section{B. Mean velocity profiles}

The dimensionless components of the mean velocity obtained from PIV measurements and the corresponding computational values are plotted in Figs. 3(a) and 3(b) as a function of the dimensionless distance $\tilde{z}$.

The streamwise velocity profile $\langle\tilde{u}\rangle(\tilde{z})$ is antisymmetric with respect to $\tilde{z}=0$ and nearly linear up to $|z| \simeq 0.3$ [see the linear fit of the numerical data shown with a dotted line in Fig. 3(a)]. For $|\tilde{z}| \simeq 0.36,\langle\tilde{u}\rangle$ reaches extrema about \pm 0.4 , the computational value $[ \pm 0.42( \pm 0.05)]$ being slightly larger than its experimental counterpart $[ \pm 0.37( \pm 0.04)]$. This slight difference is smaller than the variability of the results between distinct realizations (vertical bars) so that we do not regard it as significant. Beyond the extrema $(|\tilde{z}|>0.36),|\langle\widetilde{u}\rangle|$ decreases rapidly in order to satisfy the no-slip boundary condition on the lateral wall.

Spatial information about the structure of the flow in the tube cross section is provided by the DNS: Fig. 4(a) displays isocontours of $\langle\widetilde{u}\rangle$. This map shows that in the central part of the section (say, $|\bar{z}| \lesssim 0.3$ ), the mean axial velocity is almost independent of the spanwise coordinate $y$.

The crosswise mean velocity component $\langle\widetilde{w}\rangle$ is plotted in Fig. 3(b) as a function of $\widetilde{z}$. This component is observed to be about 30-40 times lower than $|\langle\tilde{u}\rangle|$ at the same $|\tilde{z}|$. Moreover, the sign of $\langle\widetilde{w}\rangle$ and its variation with $\tilde{z}$ are found to behave similarly in all experiments as well as in the numerical simu- 

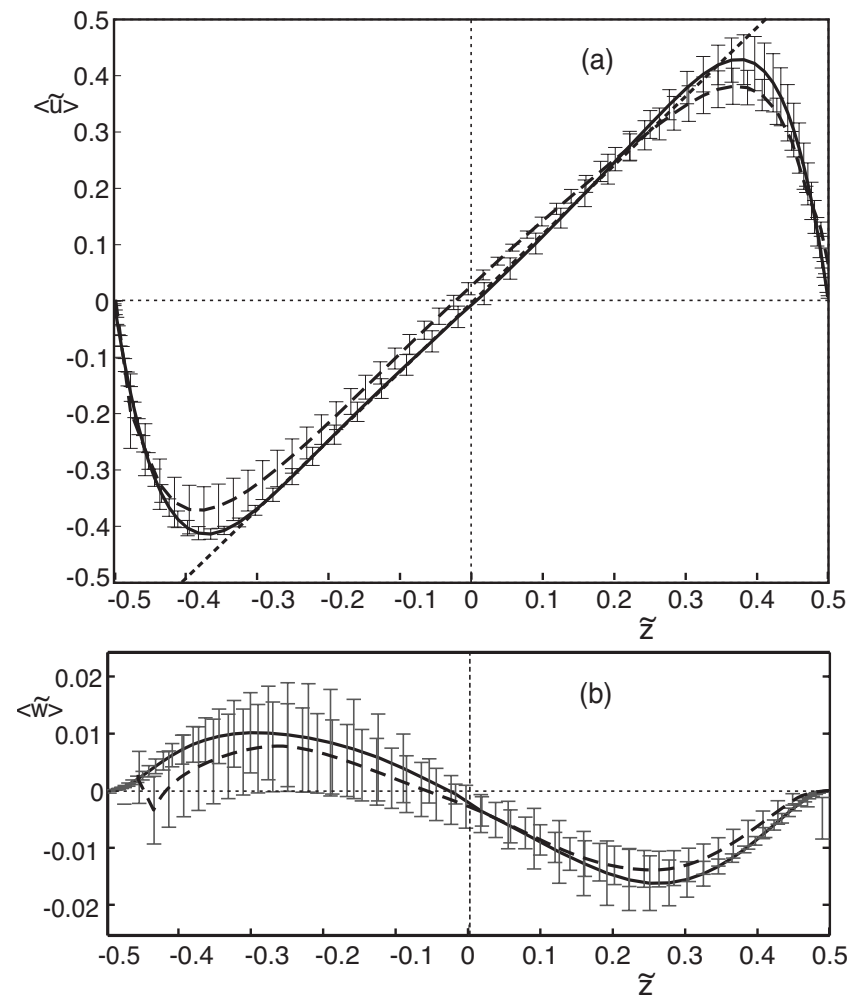

FIG. 3. Dimensionless average velocity components as a function of the crosswise distance $\tilde{z}$ from the axis. (a) Streamwise component: $\langle\tilde{u}\rangle=\langle u\rangle / V_{t}$; (b) transverse component: $\langle\widetilde{w}\rangle=\langle w\rangle / V_{t}$. Experimental data: dashed lines; DNS data: solid lines; linear fit of the variation of $\langle\widetilde{u}\rangle$ with $\widetilde{z}$ in the central part of the flow (DNS data): dotted line. The meaning of the error bars is the same as in Fig. 2

lation. Although it is much smaller than that of the axial velocity $\langle\widetilde{u}\rangle$, the nonzero value of $\langle\widetilde{w}\rangle$ is therefore a true structural feature of the flow and does not result from the variability of the results.

More information on $\langle\widetilde{w}\rangle$ and on the three-dimensional structure of the flow is provided in Fig. 4(b) where the distribution of the mean velocity vectors in the $y z$-plane and that of the associated streamwise vorticity component $\left\langle\omega_{x}\right\rangle$ obtained from the DNS are displayed. Four persistent counter-rotating structures associated with a nonzero mean component of the streamwise vorticity are observed in the cross section of the tube. The characteristic velocity of this secondary flow is about $3 \%$ of the primary velocity $\langle\widetilde{u}\rangle$. The expected left-right and top-bottom symmetries are only approximately satisfied: This suggests an incomplete convergence of the averages due to the relatively short physical time lapse spanned by the numerical data.

Since the present flow is nearly parallel to $x$, the components of the vorticity in the $y, z$ plane are given by $\omega_{y}=\partial u / \partial z$ and $\omega_{z}=-\partial u / \partial y$. This is formally analogous to the relation $\boldsymbol{v}=\nabla \wedge \psi$ between a $2 D$ incompressible velocity field $\boldsymbol{v}$ and the corresponding streamfunction $\psi$. The axial velocity component $u$ represents therefore a "streamfunction" for the vorticity field and the isovelocity contours [Fig. 4(a)] are also the vortex lines. Therefore, in the present case, the vortex lines form anticlockwise loops about the maximum velocity in the upper half of the tube, and clockwise loops in (a)

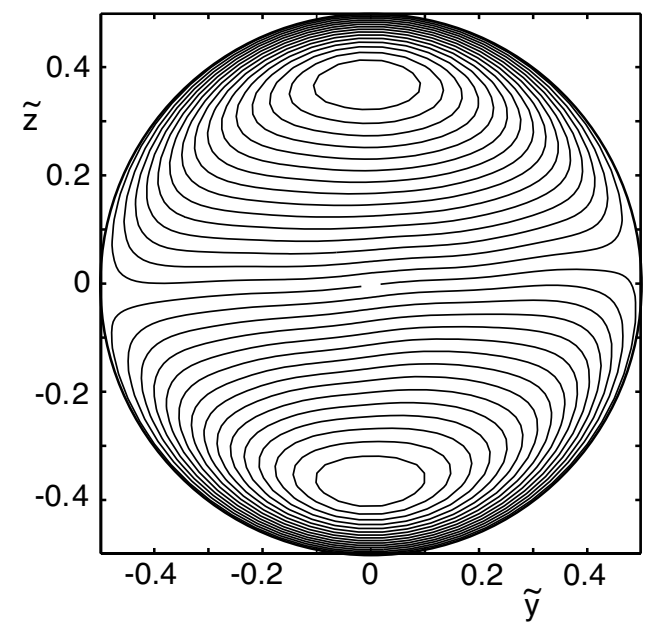

(b)

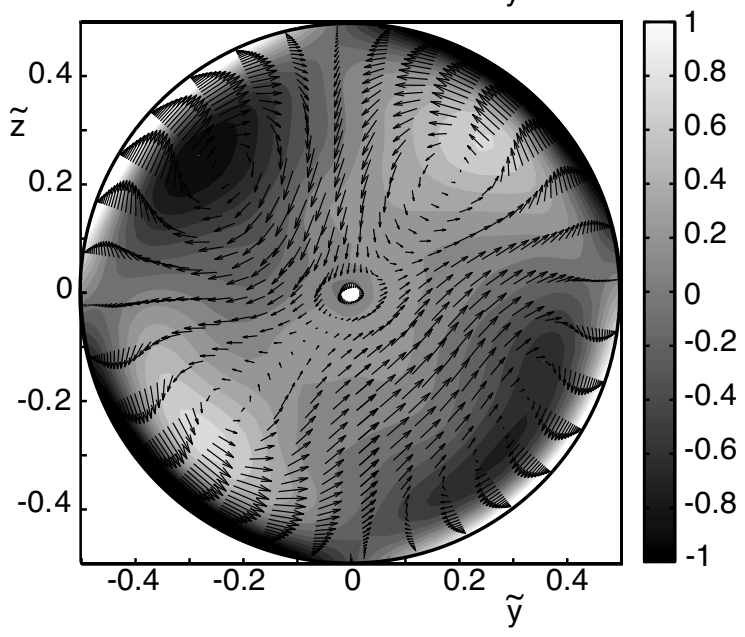

FIG. 4. DNS of the spatial distribution of the mean flow in the tube cross section. (a) Isovalues of the axial mean velocity component $\langle\tilde{u}\rangle$. The corresponding values of $\langle\tilde{u}\rangle$ range from -0.4 (smallest loop in the bottom part) to 0.4 (smallest loop in the upper part). (b) Secondary velocity field in the tube cross section superimposed on the magnitude of the streamwise vorticity $\left\langle\omega_{x}\right\rangle$ (the vorticity scale at the right is in dimensionless units).

the lower half. The velocity gradient, and hence the vorticity, reaches its highest magnitude near the lateral wall.

Because the vortex lines are isovelocity contours, there is no tilting mechanism in the base flow capable of producing a streamwise component of the vorticity. Thus the generation of the secondary flow has to be sought in the turbulent fluctuations. Consider a fluctuation in the first $(y, z)$ quadrant, say near $\tilde{y}=0.3$ and $\tilde{z}=0.3$, which exchanges a small line element of high vorticity located near the wall with another element of lower vorticity further away from the wall. We assume that this exchange does not modify the orientation of the line element in the tube cross section. Because of the closer spacing of the isovelocity lines near the top of the tube, the high vorticity line that has moved away from the wall will find its top end in a higher mean flow than its lower end. The mean flow will therefore tilt the vorticity fluctuation toward the axial direction, thus creating a counterclockwise secondary motion in the first quadrant. Applying the same argument in the other three quadrants leads to the four-cell structure revealed by Fig. 4(b) with the expected sign.

The key ingredient required in the above mechanism is 


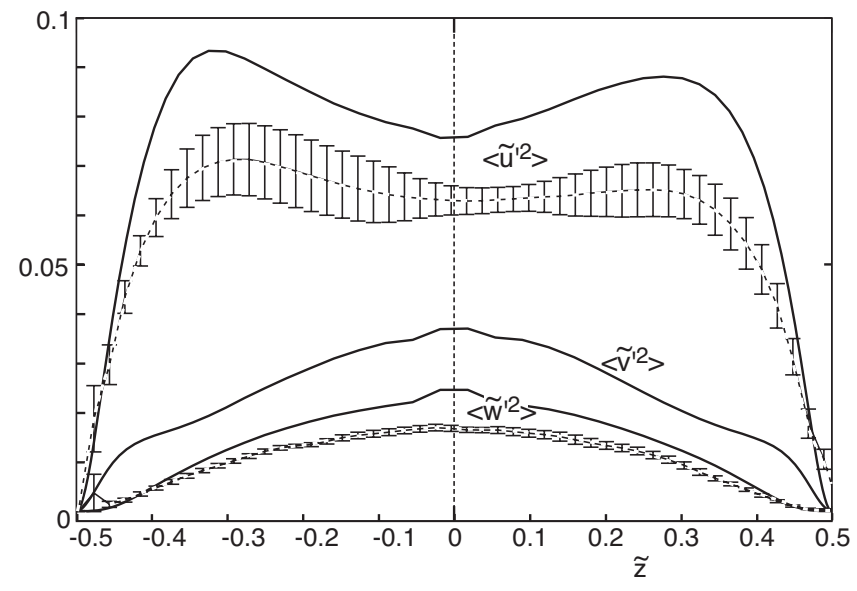

FIG. 5. Normalized mean square components of the velocity fluctuations as a function of $\widetilde{z}:\left\langle\widetilde{u}^{\prime 2}\right\rangle$ (top two curves), $\left\langle\widetilde{w}^{\prime 2}\right\rangle$ (bottom two curves), and $\left\langle\widetilde{v}^{\prime 2}\right\rangle$ (central curve). Dashed lines: experimental data; solid lines: computational results ( $v^{\prime}$ could only be determined from the computations).

the variation of the spacing of the isovelocity contours (or, equivalently, the variation of the vorticity) along the wall surface. Therefore, this mechanism would not produce any secondary flow in an axisymmetric Poiseuille flow in a circular duct. On the other hand, it holds in a square duct and may explain the formation of the eight-cell secondary structure classically observed near the duct corners. ${ }^{16-19}$

Note that this simple tilting mechanism accounts for the appearance of a secondary flow without involving buoyancy effects. The main limitation of the above argument is that it is based on a linear inviscid approach. Therefore there is no way at this stage to determine whether the streamwise vorticity fluctuation it generates will actually be amplified or rather damped by nonlinear and viscous effects. This would require a systematic characterization of the inhomogeneities of the turbulence field, i.e., of the components of the Reynolds stress tensor, which is beyond the scope of the present work. This analysis is carried out in another paper. ${ }^{20}$ It shows that the Reynolds number stresses inhomogeneity resulting from the combined effect of the mean shear and the wall results in a source term in the mean streamwise vorticity balance, which turns out to sustain the formation of the observed secondary mean motion.

\section{Turbulent intensities}

Another important feature of the flow is the turbulent intensity which may be characterized by the mean squares of the three fluctuating velocity components. The values $\left\langle\tilde{u}^{\prime 2}\right\rangle$ and $\left\langle\widetilde{w}^{\prime 2}\right\rangle$ (normalized by $V_{t}^{2}$ ) of the streamwise and crosswise components that could be determined experimentally are plotted in Fig. 5 together with the computational values obtained for all three components.

One may first observe that the experimental and computational values of $\left\langle\tilde{u}^{\prime 2}\right\rangle$ and $\left\langle\tilde{w}^{\prime 2}\right\rangle$ behave very similarly. Both approaches provide values of $\left\langle\tilde{u}^{\prime 2}\right\rangle$ three to four times higher than those of $\left\langle\widetilde{w}^{\prime 2}\right\rangle$ at all $\tilde{z}$ values. They also indicate the

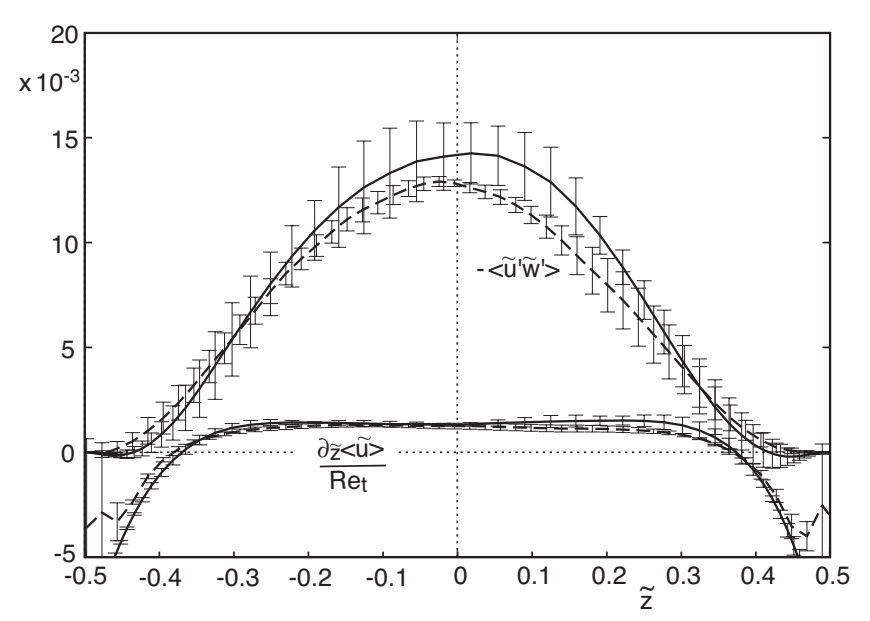

FIG. 6. Dimensionless viscous and turbulent stress components $\operatorname{Re}_{t}^{-1} \partial_{\bar{z}}\langle\tilde{u}\rangle$ (bottom curves) and $-\left\langle\tilde{u}^{\prime} \tilde{w}^{\prime}\right\rangle$ (top curves) as a function of $\tilde{z}$. Solid lines: computation; dashed lines: experiments.

same type of profiles, i.e., a $\left\langle\tilde{u}^{\prime 2}\right\rangle$ profile with two maxima at $\tilde{z} \simeq \pm 0.3$ and a broad central minimum, and a $\left\langle\widetilde{w}^{\prime 2}\right\rangle$ profile with a single central maximum. The spanwise intensity $\left\langle\tilde{v}^{\prime 2}\right\rangle$ could only be determined from the computations. Its profile is similar to that of $\left\langle\widetilde{w}^{\prime 2}\right\rangle$ but its magnitude is found to be about $50 \%$ larger.

The above three profiles make it clear that the turbulent field is strongly anisotropic in the core of the flow where the lateral wall is expected to have little effect. Therefore, the origin of this anisotropy is likely the presence of the mean shear. Indeed, similar anisotropies have been consistently reported in turbulent shear flows. ${ }^{21-23}$ This anisotropy reflects the fact that the energy transfer from the mean flow toward the turbulent fluctuations essentially feeds the streamwise component $u^{\prime}$. This energy is then redistributed onto the other two components $v^{\prime}$ and $w^{\prime}$, thanks to pressure fluctuations but this redistribution is uneven, owing to the role of the mean shear which favors the spanwise component. For a homogeneous turbulent shear flow, Tavoularis and Karnik ${ }^{23}$ reported $\left\langle\tilde{w}^{\prime 2}\right\rangle /\left\langle\tilde{u}^{\prime 2}\right\rangle \simeq 0.43$ and $\left\langle\tilde{v}^{\prime 2}\right\rangle /\left\langle\tilde{u}^{\prime 2}\right\rangle \simeq 0.53$, whereas the present results indicate values of these ratios taken at $\tilde{z}=0$ about 0.30 and 0.46 , respectively. Similarly, these authors reported $\left\langle\widetilde{u}^{\prime} \widetilde{w}^{\prime}\right\rangle /\left\langle\widetilde{u}^{\prime 2}\right\rangle \simeq-0.32$, whereas the maximum of the turbulent stress reported in Fig. 6 below leads to $\left\langle\tilde{u}^{\prime} \tilde{w}^{\prime}\right\rangle /\left\langle\tilde{u}^{\prime 2}\right\rangle \simeq-0.20$. Therefore, the present turbulent field is found to be slightly more anisotropic than that in a homogeneous shear flow but the general tendencies are similar.

Note that the maximum of $\left\langle\tilde{u}^{\prime 2}\right\rangle$ located at $|\tilde{z}| \simeq 0.3$ is probably due to a "double wall effect." On the one hand, the near-wall shear rate is higher than that in the core so that there is a stronger source of $u^{\prime}$ near the wall; on the other hand, the image effect of the wall is known to inhibit the redistribution mechanism toward the $v^{\prime}$ and $w^{\prime}$ components. Both effects tend to increase $u^{\prime}$ at the expense of the other two components, thus resulting in a more "one-component" turbulence near the wall. 


\section{MOMENTUM TRANSPORT IN THE MIXING FLOW}

\section{A. Governing equation}

We now turn to the mean momentum balance in the mixing flow. Following the considerations developed in Secs. II C and III A, we disregard derivatives with respect to $x$ and $t$ so that the $x$-component of the Reynolds equation may be written as

$$
\begin{aligned}
\langle\rho(0)\rangle & \left(\langle v\rangle \partial_{y}\langle u\rangle+\langle w\rangle \partial_{z}\langle u\rangle+\partial_{y}\left\langle u^{\prime} v^{\prime}\right\rangle+\partial_{z}\left\langle u^{\prime} w^{\prime}\right\rangle\right) \\
= & -\partial_{x}\langle p\rangle-[\langle\rho(0)\rangle+\delta \rho(z)] g \cos \theta \\
& +\langle\rho(0)\rangle \nu\left(\partial_{z^{2}}\langle u\rangle+\partial_{y^{2}}\langle u\rangle\right) .
\end{aligned}
$$

In spite of the circular geometry of the tube section, Cartesian coordinates are used in the equation both because the measurement is achieved in the $(x, z)$ plane and because transverse gravity oriented along the $z$ axis breaks the axisymmetry of the flow.

As mentioned in Sec. III A, the cross-sectional average $\langle\rho\rangle_{y, z} g \cos \theta \approx\langle\rho(\widetilde{z}=0)\rangle g \cos \theta$ of the hydrostatic contribution is balanced by the axial pressure gradient $\partial_{x}\langle p\rangle$ so that these two terms can be removed. Using the normalization discussed in Sec. II C and introducing the dimensionless variable $\delta \widetilde{\rho}(\widetilde{z})=\delta \rho(z) /\langle\rho(0)\rangle$, Eq. (2) becomes

$$
\begin{aligned}
\delta \widetilde{\rho}(\widetilde{z}) \frac{\cos \theta}{\mathrm{At}}= & -\langle\widetilde{v}\rangle \partial_{\tilde{y}}\langle\widetilde{u}\rangle-\langle\widetilde{w}\rangle \partial_{\tilde{z}}\langle\widetilde{u}\rangle-\partial_{\tilde{y}}\left\langle\widetilde{u}^{\prime} \widetilde{v}^{\prime}\right\rangle-\partial_{\tilde{z}}\left\langle\widetilde{u}^{\prime} \widetilde{w}^{\prime}\right\rangle \\
& +\operatorname{Re}_{t}^{-1}\left(\partial_{\tilde{z}}\langle\widetilde{u}\rangle+\partial_{\tilde{y}}\langle\widetilde{u}\rangle\right) .
\end{aligned}
$$

The buoyancy term on the left-hand side is the driving force of the local flow, whereas the terms on the right-hand side represent advection of $\langle\tilde{u}\rangle$ by the mean secondary flow, turbulent transport, and viscous diffusion within the cross section, respectively.

\section{B. Turbulent and viscous stresses}

In this section, we compare the dimensionless in-plane stress components $\operatorname{Re}_{t}^{-1} \partial\langle\widetilde{u}\rangle / \partial \widetilde{z}$ and $-\left\langle\widetilde{u}^{\prime} \widetilde{w}^{\prime}\right\rangle$ whose net flux in the $z$-direction provides the crosswise transport of the mean streamwise momentum by viscosity and by turbulent fluctuations, respectively. As pointed out in Sec. II D, the in-plane terms are the only ones which could be measured experimentally; in a two-dimensional mean flow taking place in the $x z$-plane, they would also be the only stress components involved in the mean momentum balance.

The variations of the above two in-plane terms in the $\tilde{z}$-direction are plotted in Fig. 6 . The Reynolds stress $-\left\langle\widetilde{u}^{\prime} \widetilde{w}^{\prime}\right\rangle$ reaches a maximum on the tube axis and decreases gradually to zero at the wall. In contrast, the viscous stress changes sign for $|\bar{z}| \approx 0.36$ and becomes much larger than the turbulent stress near the wall, whereas it is much smaller (by up to a factor of 10 at $|\vec{z}|=0$ ) throughout the central $70 \%$ of the tube. Because of this change in sign of the viscous stress, the global stress vanishes in the vicinity of the mean velocity extrema. Again, the computational and experimental determinations of these stresses are in good agreement; the values of the Reynolds stress they provide coincide within the variability of the experimental values. The uncertainty on the computational prediction is larger, owing to the shorter averaging time and to the availability of only one "numerical experiment."

By comparing Figs. 5 and 6 one also notices that the orders of magnitude of $\left\langle\widetilde{w}^{\prime 2}\right\rangle$ and $-\left\langle\widetilde{u}^{\prime} \widetilde{w}^{\prime}\right\rangle$ are similar in the core region but that both are significantly smaller than $\left\langle\widetilde{u}^{\prime 2}\right\rangle$. This implies that only a small part of the streamwise fluctuation $u^{\prime}$ is correlated with $w^{\prime}$, a usual feature of turbulent shear flows.

In terms of momentum transfer, the presence of two particular locations, say $\tilde{z}= \pm \tilde{z}_{m}$, where the total stress $\operatorname{Re}_{t}^{-1} \partial_{\bar{z}}\langle\widetilde{u}\rangle-\left\langle\widetilde{u}^{\prime} \widetilde{w}^{\prime}\right\rangle$ vanishes makes it possible to identify three different regions in the flow, namely, a core region and two near-wall regions. Since the total stress is zero at $\tilde{z}= \pm \widetilde{z}_{m}$, the core region located within these two locations does not exchange any momentum with the near-wall regions $\left(\widetilde{z}_{m}<|\tilde{z}|<1 / 2\right)$. This is because the integral of the buoyancy force throughout the core is zero, although buoyancy is obviously locally nonzero and drives the local flow field. In the upper part of this core region $(|z|>0)$, the fluid receives momentum from the positive buoyancy force, which results in a net downward momentum flux through the plane $\widetilde{z}=0$. Then, in the lower region $\tilde{z}<0$, the buoyancy force is negative and the fluid loses the momentum that enters through the plane $\tilde{z}=0$.

In each near-wall region, the wall shear stress is nonzero so that there is a net momentum transfer between the fluid and the wall. The upper near-wall region transfers momentum to the wall because the local buoyancy force is positive throughout, whereas the reverse is true for the lower nearwall region so that momentum enters the fluid at the lower wall. The above picture drastically differs from that which holds in a usual channel flow where momentum is transferred from the core to both walls because the external force (i.e., the streamwise pressure gradient) is constant throughout the flow.

The most classical turbulence closure by which the Reynolds stress $-\left\langle\widetilde{u}^{\prime} \widetilde{w}^{\prime}\right\rangle$ may be related to the mean velocity gradient $\partial_{z}\langle\widetilde{u}\rangle(\tilde{z})$ is the mixing length model which expresses this "rheological" relation in the form

$$
-\left\langle u^{\prime} w^{\prime}\right\rangle=l_{m}^{2}(z)\left\langle\partial_{z} u\right\rangle\left|\left\langle\partial_{z} u\right\rangle\right|
$$

where $l_{m}$ is the mixing length. This amounts to introducing a turbulent viscosity $\nu_{t}=l_{m} u^{*}$ in which the turbulent velocity scale $u^{*}$ is assumed to be $u^{*}=l_{m}\left|\left\langle\partial_{z} u\right\rangle\right|$. The variations of $\tilde{l}_{m}=l_{m} / d$ with $\tilde{z}$ derived from both the experiments and the computations are displayed in Fig. 7 . For $-0.35 \leq \widetilde{z} \leq 0.35, \tilde{l}_{m}$ only exhibits small variations and remains in the range $0.07 \leq \tilde{l}_{m} \leq 0.11$. However, owing to Eq. (4), $\tilde{l}_{m}$ cannot be expected to remain strictly constant throughout the core since $\left\langle\widetilde{u}^{\prime} \widetilde{w}^{\prime}\right\rangle$ varies with $\widetilde{z}$ while $\partial_{\tilde{z}}\langle\tilde{u}\rangle$ is almost constant.

In the region where $\partial_{\bar{z}}\langle\tilde{u}\rangle$ is negative $(|\bar{z}| \geq 0.36)$, $-\left\langle\widetilde{u}^{\prime} \widetilde{w}^{\prime}\right\rangle$ and $\partial_{\bar{z}}\langle\widetilde{u}\rangle$ have opposite signs so that the mixing length model is unphysical and no values of $\tilde{l}_{m}$ are displayed.

The nearly constant mixing length in the core of the flow compares well with the recent experiments by Odier et al., ${ }^{2}$ although the geometry and the confinement significantly dif- 


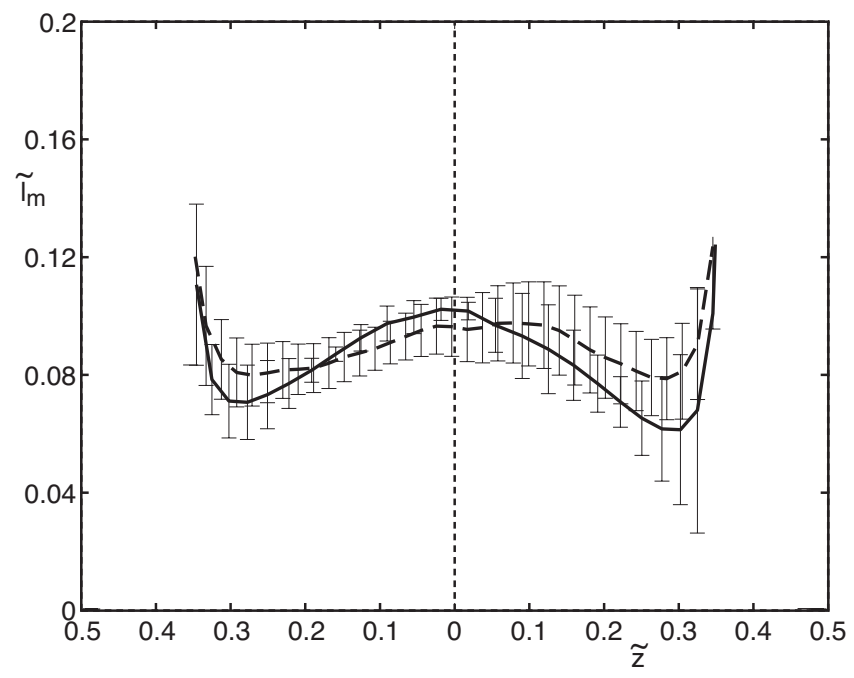

FIG. 7. Normalized mixing length $\tilde{l}_{m}$ as a function of $\tilde{z}$. Solid line: computation; dashed lines: experiments.

fer from ours. This slow variation of $\tilde{l}_{m}$ is also reminiscent of free shear flows such as jets and mixing layers where $\tilde{l}_{m}$ is known to be almost constant, at odds from usual channel or pipe flows where $\widetilde{l}_{m}$ increases linearly with the distance to the wall. Obviously, these remarkably contrasted behaviors are directly related to the structural difference between the ways momentum is transferred in the core of the present flow as compared to the case of usual wall-bounded flows.

The above analysis was carried out with the implicit assumption that the flow is invariant in the spanwise direction. This assumption may actually be checked quantitatively using the DNS results. The corresponding distribution of the turbulent stress $-\left\langle\widetilde{u}^{\prime} \widetilde{w}^{\prime}\right\rangle$ in the tube cross section is displayed in Fig. 8(a). For $|\vec{z}| \leq 0.3$, the values of $-\left\langle\widetilde{u}^{\prime} \widetilde{w}^{\prime}\right\rangle$ only weakly depend on $y$, except near the walls where they steeply decrease. The isocontours of $\langle\tilde{u}\rangle$ in Fig. 4 are also almost parallel in the same region. Therefore the viscous term $\operatorname{Re}_{t}^{-1} \partial\langle\widetilde{u}\rangle / \partial \widetilde{z}$ is nearly independent of $y$ up to the vicinity of the walls. This shows that the results obtained in the vertical midplane $y=0$ are actually valid over a significant range of spanwise locations and supports the use of a twodimensional approximation to obtain a first-order description of the mean momentum transfer in the flow.

A final important issue is the relative influence of transport terms involving the out-of-plane (i.e., spanwise) velocity component which, too, can only be determined from DNS results. Figure $8(\mathrm{~b})$ displays the variations of $-\left\langle\tilde{u}^{\prime} \tilde{v}^{\prime}\right\rangle$ in the flow section. The left-right symmetry of the averaged flow requires that $-\left\langle\widetilde{u}^{\prime} \widetilde{v}^{\prime}\right\rangle=0$ for $y=0$. This symmetry condition is not exactly satisfied in Fig. 8(b) because of the marginal convergence of the averaging process but the value on the plane $y=0$ is always less than $1 / 15$ that of $-\left\langle\widetilde{u}^{\prime} \widetilde{w}^{\prime}\right\rangle$. Outside this plane, $-\left\langle\tilde{u}^{\prime} \widetilde{v}^{\prime}\right\rangle$ is approximately an odd function of both $z$ and $y$ and reaches extrema whose absolute value is up to $1 / 3$ that of $-\left\langle\tilde{u}^{\prime} \widetilde{w}^{\prime}\right\rangle$. It will be seen below that this turbulent stress has a significant contribution to the momentum balance. (a)
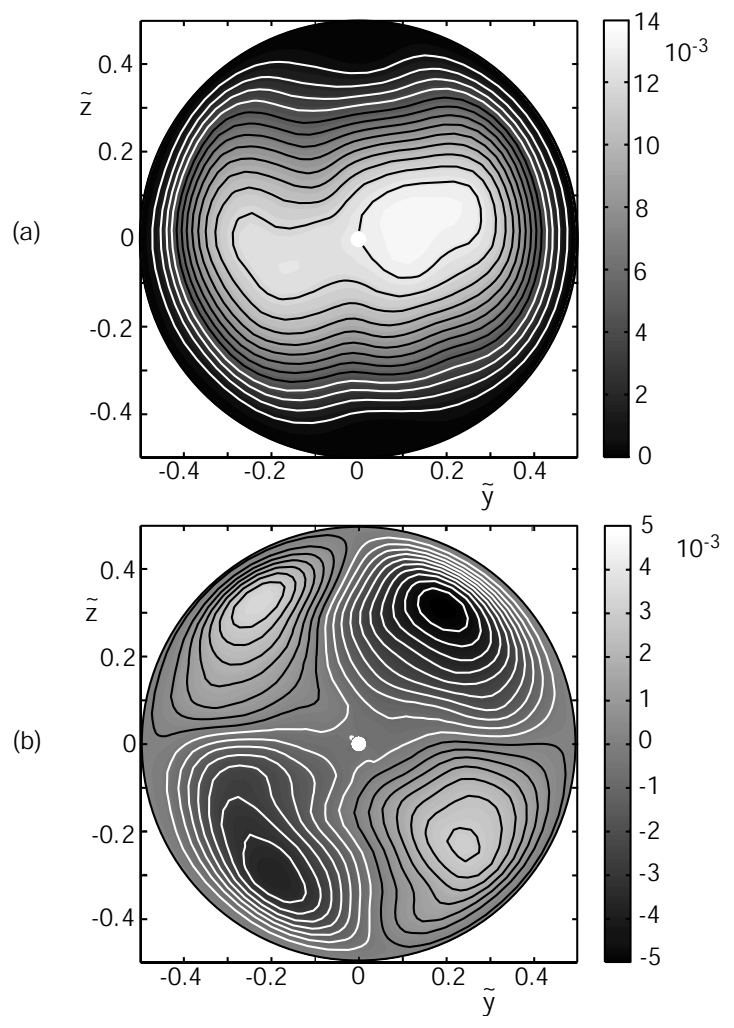

FIG. 8. Gray scale maps of the turbulent stresses: (a) $-\left\langle\widetilde{u}^{\prime} \widetilde{w}^{\prime}\right\rangle$ and (b) $-\left\langle\widetilde{u}^{\prime} \widetilde{v}^{\prime}\right\rangle$ in the tube cross section (DNS results). The scales at the right correspond to the values of the respective components. The isocontour separation is $10^{-3}$ in (a) and $5 \times 10^{-4}$ in (b).

\section{MOMENTUM BALANCE IN THE MIXING FLOW}

For a purely two-dimensional flow with only a streamwise nonzero mean velocity (i.e., with $\langle\widetilde{w}\rangle=0$ ), the momentum input provided by the buoyancy forces would be strictly balanced by the combination of the two in-plane viscous and turbulent contributions discussed above. However, the present flow is actually three-dimensional and other contributions may play a role in the averaged streamwise momentum balance. This local momentum balance is expressed by Eq. (3). The various contributions to the net momentum transport contained in its right-hand side are plotted in the main graphs of Figs. 9(a) and 9(b) as a function of the normalized distance $\widetilde{z}$. Various combinations of these terms are compared to the buoyancy force in the insets.

In Figs. 9(a) and 9(b), the two in-plane terms $\operatorname{Re}_{t}^{-1} \partial_{z_{z}}\langle\widetilde{u}\rangle$ and $-\partial_{\bar{z}}\left\langle\widetilde{u}^{\prime} \widetilde{w}^{\prime}\right\rangle$ are those already discussed in Sec. IV B. The third in-plane term $-\langle\widetilde{w}\rangle \partial_{\tilde{z}}\langle\widetilde{u}\rangle$ corresponds to the transport of the mean velocity gradient in the $z$-direction by the crosswise secondary mean velocity $\langle\widetilde{w}\rangle$ discussed in Sec. III B. Although the four-cell recirculation flow discussed in Sec. III B is weak, its contribution to the momentum balance is not negligible [dashed-dotted line in the main graphs of Figs. 9(a) and 9(b)]; the strong mean shear $\partial_{\tilde{z}}\langle\tilde{u}\rangle$ compensates indeed for the small value of the secondary velocity $\langle\widetilde{w}\rangle$. Its dependence on the distance to the axis is similar to that of $-\partial_{\bar{z}}\left\langle\widetilde{u}^{\prime} \widetilde{w}^{\prime}\right\rangle$ but it is smaller by a factor of more than 2 and of opposite sign.

Out-of-plane terms could only be determined from the 

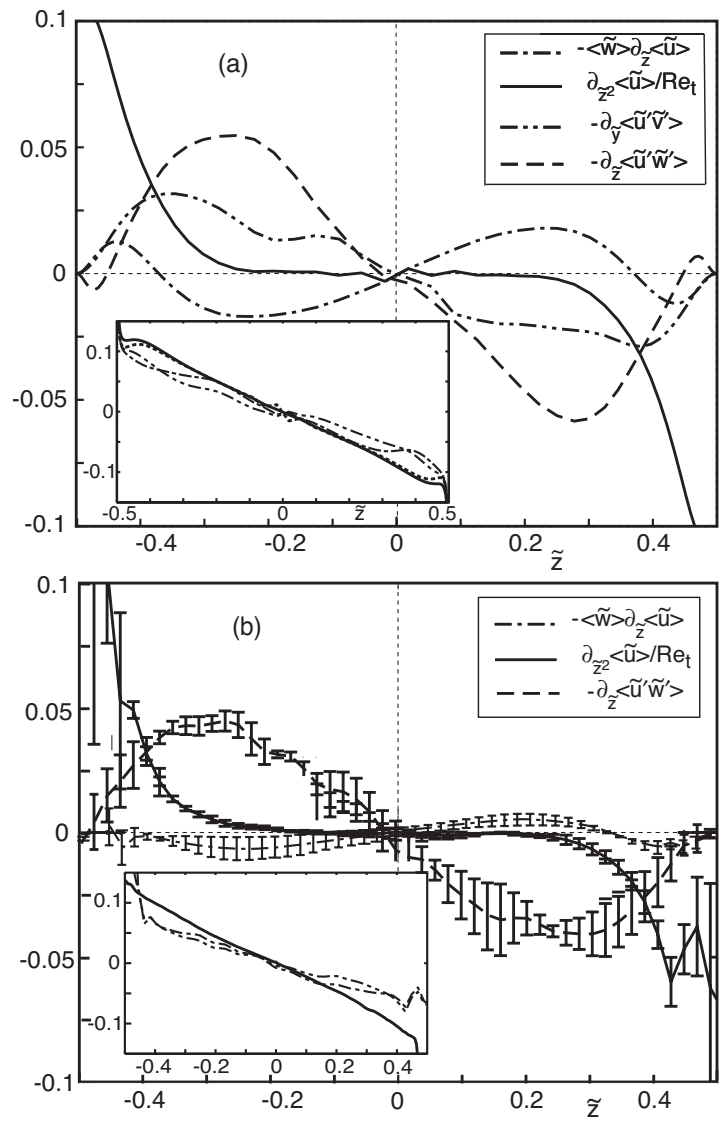

FIG. 9. Normalized transport terms of Eq. (3) as a function of $\tilde{z}$ : (a) computational results; (b) experimental data. Insets: compared z-profiles of the buoyancy force (solid line) and of various combinations of the transport terms (dashed-dotted line: sum of viscous and turbulent terms $\operatorname{Re}_{t}^{-1} \partial_{z^{2}}\langle\tilde{u}\rangle$ $-\partial_{z}\left\langle u^{\prime} w^{\prime}\right\rangle$; dashed-dotted-dotted line: same as the previous sum plus the contribution $\left\langle\widetilde{w} \partial_{z}\langle\tilde{u}\rangle\right.$; dotted line: sum of all momentum transport terms [only in Fig. 9(a)]).

DNS results. As already seen in Sec. IV B, $\left\langle\widetilde{u}^{\prime} \widetilde{v}^{\prime}\right\rangle$ is an odd function of $y$ so that $-\partial_{\tilde{y}}\left\langle\widetilde{u}^{\prime} \widetilde{v}^{\prime}\right\rangle$ is nonzero in the vertical diametrical plane. The magnitude of its extrema is about half that of the in-plane turbulent contribution but occurs at a larger distance from the axis, say $|\tilde{z}| \simeq 0.35$ [dashed-dotted line in the main graph of Fig. 9(b)]. The out-of-plane term associated with the spanwise component of the secondary mean flow is almost zero, as expected from the left-right symmetry of the flow, while the viscous term $\partial_{\tilde{y}}\langle\tilde{u}\rangle / \operatorname{Re}_{t}$ is one order of magnitude lower than all in-plane contributions and is therefore neglected in the following analysis. Note that the low value of the latter term is in agreement with the observation that the streamwise velocity profile is almost invariant along the spanwise direction in the central part of the tube (Fig. 4), like in a turbulent channel flow.

The inset of Fig. 9(a) compares the profile of the sum of all four in-plane and out-of-plane transport terms (dotted line) with that of the buoyancy force (solid line). The excellent collapse of the two profiles confirms that all relevant contributions have been taken into account. In particular, this collapse completely supports our initial claim that there is a local equilibrium between the local buoyancy force $\delta \rho(z) g \cos \theta$ and the various contributions to the momentum transfer provided by the $y$ - and $z$-derivatives of the viscous and turbulent stresses. In other words, it validates the idea that derivatives with respect to $x$ and $t$ are negligible in Eq. (3).

Experimentally, only the in-plane transport terms could be measured in the vertical midplane $y=0$. One can then wonder to which extent the momentum balance would be satisfied by considering only the experimentally measurable terms, as discussed in Sec. IV B. The sum $\operatorname{Re}_{t}^{-1} \partial_{\tilde{z}}\langle\tilde{u}\rangle$ $-\partial_{\bar{z}}\left\langle\widetilde{u}^{\prime} \widetilde{w}^{\prime}\right\rangle$ is plotted as a function of $\widetilde{z}$ in the insets of Figs. 9(a) and 9(b) (dashed-dotted line). For both experiments and computations, the agreement with the local value of the buoyancy force is reasonable in the core of the flow $(|z| \leq 0.25)$ but the relative difference increases up to $30 \%$ for $\tilde{z}= \pm 0.4$. Including the contribution of the secondary flow $-\langle\widetilde{w}\rangle \partial_{\tilde{z}}\langle\widetilde{u}\rangle$ does not improve the agreement (dasheddotted-dotted line in the insets) since its sign is opposite to that of the main turbulent contribution.

To conclude, both the experimental and the computational results indicate that the two-dimensional approximation provides a reasonable first-order description of the momentum transfer processes in the flow close to the plane $\tilde{z}=0$. In contrast, three-dimensional effects which arise both through the contribution of the mean secondary flow and from that of the out-of-plane turbulent stress $-\left\langle\widetilde{u}^{\prime} \widetilde{v}^{\prime}\right\rangle$ are required to obtain a quantitative description of the outer region located further away from $\widetilde{z}=0$.

\section{DISCUSSION AND CONCLUSION}

The experiments and computations reported here provide a global picture of the flow field and momentum exchange in the turbulent buoyancy-driven flow that takes place in a long tilted tube filled with two fluids of slightly different densities. In contrast to pressure-driven turbulent pipe and channel flows, the momentum exchange in the core turbulent region $(|z| \leq 0.36)$ only involves the denser and lighter regions of the core with no momentum transfer between this core and the lateral wall. In this region, the mean concentration and axial velocity vary linearly with the distance $|z|$ to the axis, this linear region extending even closer to the wall for the concentration. In some respects, the flow in the core appears as a free shear flow driven by the buoyancy force. The mean flow is nearly parallel to the tube axis but a small secondary mean motion made of four counter-rotating cells that fill the entire cross section also exists, owing to the combined effect of the nonaxisymmetric mean shear and of the circular geometry of the pipe. Although the magnitude of this secondary motion is only about $3 \%-4 \%$ that of the maximum axial velocity, its contribution to the transfer of momentum is not negligible.

The turbulent field exhibits a marked anisotropy. More precisely, the streamwise intensity $\left\langle\widetilde{u}^{\prime 2}\right\rangle$ is roughly two to three times larger than the other two intensities. It reaches its maximum away from the axis, close to $|\bar{z}| \simeq 0.3$, a common feature of near-wall flows where wall effects are known to result in a pronounced maximum of the streamwise fluctuation. The other two intensities reach their maximum on the tube axis. The spanwise intensity $\left\langle\widetilde{v}^{\prime 2}\right\rangle$ is about $50 \%$ larger than the crosswise one $\left\langle\widetilde{w}^{\prime 2}\right\rangle$. Both the magnitude of the in- 
plane turbulent stress $\left\langle\widetilde{u}^{\prime} \widetilde{w}^{\prime}\right\rangle$ and its dependence with respect to $\widetilde{z}$ are similar to those of $\left\langle\widetilde{w}^{\prime 2}\right\rangle$, its magnitude being only about $20 \%$ that of $\left\langle\widetilde{u}^{\prime 2}\right\rangle$. These anisotropy levels between the various components of the Reynolds stress tensor are consistent with the known characteristics of homogeneous turbulent shear flows.

A first-order picture of momentum transfer in the vertical midplane $y=0$ is provided by a two-dimensional description limited to the main turbulent shear stress $-\left\langle\widetilde{u}^{\prime} \widetilde{w}^{\prime}\right\rangle$ and to the in-plane viscous stress $\partial_{\bar{z}}\langle\tilde{u}\rangle / \operatorname{Re}_{t}$. In the core region $(|\vec{z}| \leq 0.36)$, the turbulent contribution is dominant and is about ten times larger than the viscous term close to the axis. The variation of $\left\langle\widetilde{u}^{\prime} \widetilde{w}^{\prime}\right\rangle$ with $\widetilde{z}$ is approximately accounted for by assuming a constant mixing length $l_{m} / d \simeq 0.1$. Outside the core region (i.e., for $|\vec{z}| \geq 0.36$ ), viscous momentum transport dominates. As the buoyancy force is positive (negative) close to the upper (lower) part of the wall, the fluid loses (gains) momentum at the upper (lower) wall. In a more thorough analysis, other contributions corresponding to the transport by the mean crosswise velocity $\langle w\rangle$ and by the spanwise velocity fluctuation $v^{\prime}$ are found to be significant and need to be taken into account to achieve an accurate momentum balance, especially outside the core of the flow, i.e., for $|\tilde{z}| \gtrsim 0.25$.

In a future work, a first important issue will be the dependence of these results on the tilt angle $\theta$. Indeed, increasing $\theta$ increases the influence of the transverse gravity and leads to a complete stratification at large tilt angles. The respective widths of the turbulent and viscous regions and the concentration and velocity profiles should therefore be particularly sensitive to the value of $\theta$. Varying the Atwood number At and/or the fluid viscosity is also expected to modify the relative contribution of viscous and turbulent contributions to the momentum transport.

Moreover, the dynamics of the mixing process has not been considered in the present work. In a first approach it may be characterized from the streamwise variation of the local average $\langle c(x, t)\rangle_{y, z}$ of the concentration. ${ }^{12}$ This variation takes place over characteristic distances and large times compared to those associated with the local turbulent fluctuations. In order to understand quantitatively this variation as well as that of $\langle c\rangle$ as a function of $\widetilde{z}$, we will need to consider the transport mechanisms of concentration within a tube cross section, especially those involving the correlation of velocity and concentration fluctuations.

\section{ACKNOWLEDGMENTS}

We thank C. Borget and R. Pidoux for the design and construction of the experimental setup. We are indebted to Y. Tanino for her careful reading of the manuscript and her thoughtful suggestions. We thank MENSR for the scholar- ship supporting the Ph.D. thesis of J. Znaien. This research and the postdoctoral stay of Y. Hallez have been funded by the French ANR through the project "GIMIC" under Grant No. ANR-07-BLAN-0181.

${ }^{1}$ J. E. Simpson, Gravity Currents in the Environment and the Laboratory, 2nd ed. (Cambridge University Press, Cambridge, UK, 1997).

${ }^{2}$ P. Odier, J. Chen, M. K. Rivera, and R. E. Ecke, "Fluid mixing in stratified gravity currents: The Prandtl mixing length," Phys. Rev. Lett. 102, 134504 (2009).

${ }^{3}$ M. H. I. Baird, K. Aravamudan, N. V. Rama Rao, J. Chadam, and A. P. Peirce, "Unsteady axial mixing by natural convection in a vertical column," AIChE J. 38, 1825 (1992)

${ }^{4}$ See National Technical Information Service Document No. NTIS-PB96147897 (E. E. Zukoski, "A review of flows driven by natural convection in adiabatic shafts," NIST Report No. NIST-GCR-95-679, 1995, and references therein). Copies may be ordered from National Technical Information Service, Springfield, VA.

${ }^{5}$ S. B. Dalziel, M. D. Patterson, C. P. Caulfield, and I. A. Coomaraswamy, "Mixing efficiency in high aspect-ratio Rayleigh-Taylor experiments," Phys. Fluids 20, 065106 (2008).

${ }^{6}$ T. Seon, J. P. Hulin, D. Salin, B. Perrin, and E. J. Hinch, "LIF measurements of buoyancy-driven mixing in tilted tubes," Phys. Fluids 18, 041701 (2006).

${ }^{7}$ Y. Hallez and J. Magnaudet, "Effects of channel geometry on buoyancydriven mixing," Phys. Fluids 20, 053306 (2008).

${ }^{8}$ T. Seon, J. P. Hulin, D. Salin, B. Perrin, and E. J. Hinch, "Buoyant mixing of miscible fluids in tilted tubes," Phys. Fluids 16, L103 (2004).

${ }^{9}$ D. I. H. Barr, "Densimetric exchange flows in rectangular channels," Houille Blanche 22, 619 (1967).

${ }^{10}$ J. O. Shin, S. B. Dalziel, and P. F. Linden, "Gravity currents produced by lock exchange," J. Fluid Mech. 521, 1 (2004).

${ }^{11}$ V. K. Birman, B. A. Battandier, E. Meiburg, and P. F. Linden, "Lockexchange flows in sloping channels," J. Fluid Mech. 53, 1 (2007).

${ }^{12}$ T. Seon, J. Znaien, E. J. Hinch, B. Perrin, D. Salin, and J. P. Hulin, "Front dynamics and macroscopic diffusion in buoyancy-driven mixing in a tilted tube," Phys. Fluids 19, 1 (2007).

${ }^{13}$ S. T. Zalesak, "Fully multidimensional flux-corrected transport algorithms for fluids," J. Comput. Phys. 31, 335 (1979).

${ }^{14} \mathrm{~T}$. Bonometti and S. Balachandar, "Effect of Schmidt number on the structure and propagation of density currents," Theor. Comput. Fluid Dyn. 22, 341 (2008).

${ }^{15}$ T. Seon, D. Salin, J. P. Hulin, B. Perrin, and E. J. Hinch, "Buoyancydriven front dynamics in tilted tubes," Phys. Fluids 17, 031702 (2005).

${ }^{16}$ H. Schlichting, Boundary Layer Theory (McGraw-Hill, New York, 1968), Chap. 20.

${ }^{17}$ J. P. Johnston, "Internal flows," in Turbulence, Topics in Applied Physics Vol. 12, edited by P. Bradshaw (Springer-Verlag, Berlin, 1978), p. 109.

${ }^{18}$ A. O. Demuren and W. Rodi, "Calculation of turbulence-driven secondary motion in non-circular ducts," J. Fluid Mech. 140, 189 (1984).

${ }^{19}$ P. Bradshaw, "Turbulent secondary flow," Annu. Rev. Fluid Mech. 19, 53 (1987).

${ }^{20}$ Y. Hallez and J. Magnaudet, "Turbulence-induced secondary motion in a buoyancy-driven flow in a circular pipe," Phys. Fluids 21, 081704 (2009).

${ }^{21}$ F. H. Champagne, V. G. Harris, and S. Corrsin, "Experiments on nearly homogeneous turbulent shear flow," J. Fluid Mech. 41, 81 (1970).

${ }^{22} \mathrm{~S}$. Tavoularis and S. Corrsin, "Experiments in nearly homogenous turbulent shear flow with a uniform mean temperature gradient. Part 1," J. Fluid Mech. 104, 311 (1981).

${ }^{23} \mathrm{~S}$. Tavoularis and U. Karnik, "Further experiments on the evolution of turbulent stresses and scales in uniformly sheared turbulence," J. Fluid Mech. 204, 457 (1989). 